\title{
Neoplasias quísticas mucinosas del páncreas: caso clínico y revisión de la literatura
}

\section{Clinical case of pancreatic mucinous cystic neoplasms and literature review}

\author{
Dinimo José Bolívar S, Lázaro Antonio Arango, ${ }^{2}$ Ricardo González, ${ }^{3}$ Claudia Patricia Díaz, ${ }^{4}$ Andrés Sánchez Gil. ${ }^{5}$
}

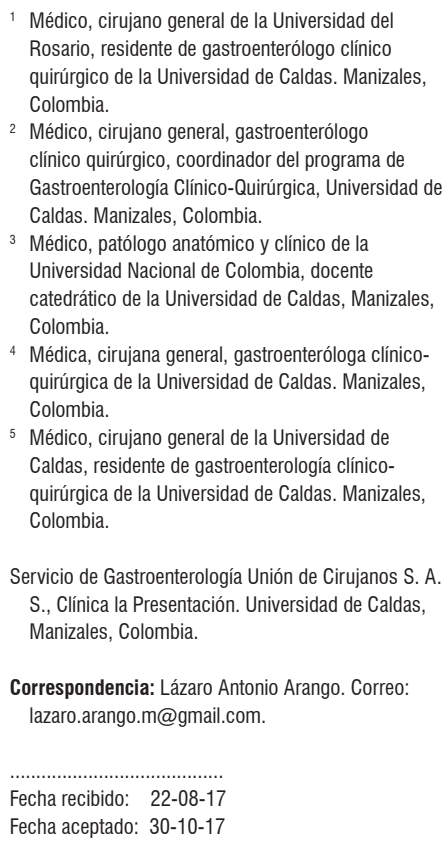

2 Médico, cirujano general, gastroenterólogo clínico quirúrgico, coordinador del programa de Gastroenterología Clínico-Quirúrgica, Universidad de Caldas. Manizales, Colombia.

3 Médico, patólogo anatómico y clínico de la Universidad Nacional de Colombia, docente catedrático de la Universidad de Caldas, Manizales, Colombia.

${ }^{4}$ Médica, cirujana general, gastroenteróloga clínicoquirúrgica de la Universidad de Caldas. Manizales, Colombia.

5 Médico, cirujano general de la Universidad de Caldas, residente de gastroenterología clínicoquirúrgica de la Universidad de Caldas. Manizales, Colombia.

Servicio de Gastroenterología Unión de Cirujanos S. A. S., Clínica la Presentación. Universidad de Caldas, Manizales, Colombia.

Correspondencia: Lázaro Antonio Arango. Correo: lazaro.arango.m@gmail.com.

Fecha recibido: $22-08-17$

Fecha aceptado: $30-10-17$

\begin{abstract}
Resumen
Las neoplasias quísticas mucinosas del páncreas son lesiones relativamente frecuentes, afectan principalmente a mujeres perimenopáusicas; la mayoría son únicos, localizados en el cuerpo y la cola del páncreas, y no tienen comunicación con el sistema ductal pancreático. Menos del $20 \%$ de los casos se asocian con malignidad. La evaluación debe incluir la presentación clínica, las imágenes, la utilización de la ultrasonografía endoscópica y toma de biopsias por punción, la citología y el análisis químico del líquido para la medición de niveles de antígeno carcinoembrionario (ACE). La resección quirúrgica completa es el único tratamiento que mejora la supervivencia a largo plazo en pacientes con lesiones quísticas mucinosas malignas. Se realiza una revisión de la literatura a propósito de un caso diagnosticado por el grupo de gastroenterología clínicoquirúrgica de la Clínica la Presentación, Manizales, Colombia.
\end{abstract}

\section{Palabras clave \\ Cistoadenoma, cistoadenocarcinoma, tumor quístico mucinoso del páncreas.}

\begin{abstract}
Mucinous cystic neoplasms of the pancreas occur relatively frequently and mainly affect women in the transition to menopause. Most of these neoplasms are unique but are located in the body and tail of the pancreas and have no communication with the pancreatic ductal system. Less than $20 \%$ are malignant. Evaluation should include clinical presentation, imaging, endoscopic ultrasonography, puncture biopsies, cytology and chemical analysis of the liquid to measure angiotensin converting enzyme (ACE) levels. Complete surgical resection is the only treatment that can improve long-term survival in patients with malignant mucinous cystic lesions. This article includes a review of the literature related to presentation of a case diagnosed by the surgical clinical gastroenterology group at Clínica la Presentación in Manizales, Colombia.
\end{abstract}

Keywords

Cystadenoma, cystadenocarcinoma, mucinous cystic tumor of the pancreas.

\section{INTRODUCCIÓN}

Los quistes pancreáticos, en su mayor proporción, son hallazgos incidentales en imágenes como la tomografía axial computarizada (TAC) o la resonancia magnética (RM). La etiología de estos quistes puede variar desde benignos, como los pseudoquistes pancreáticos y cistoadenomas serosos; hasta quistes premalignos o francamente malignos, como la neoplasia quística mucinosa o la neoplasia mucinosa papilar intraductal. El desafío clínico del 2017 fue diagnosticarlos preoperatoriamente con precisión y su potencial maligno antes llevar a cirugía, hacer vigilancia o no hacer nada (23).

Las neoplasias quísticas del páncreas pueden derivar del epitelio ductal (neoplasias quísticas serosas [NQS], neo- 
plasias quísticas mucinosas $[\mathrm{NQM}]$, neoplasias mucinosas papilares intraductales [NMPI] y neoplasias tubulares intraductales $[\mathrm{NTI}]$ ), de las células endocrinas, de las células de los ácinos pancreáticos (cistoadenoma y cistoadenocarcinoma de células acinares) y de elementos mesenquimales. Algunos tumores sólidos pseudopapilares pueden formar quistes y simular NQS o NQM (1).

La clasificación de tumores de la Organización Mundial de la Salud (OMS) de 2010 define a las NQM como una neoplasia epitelial formadora de quistes que no presenta comunicación con el conducto pancreático y que está compuesto de un epitelio columnar productor de mucina y un estroma similar al ovario (2). Aunque son menos comunes que las NQS o las NMPI, se clasifican igual que estas últimas de acuerdo con el grado de displasia (bajo, intermedio, alto y NQM asociadas con carcinoma invasor) (1). El término carcinoma in situ fue reemplazado por el de displasia de alto grado y reserva el término de carcinoma invasivo para malignidad (2). Se describe un caso clínico de una paciente con NQM y se revisa la literatura.

\section{CASO CLÍNICO}

La paciente es una mujer de 73 años remitida de un hospital de III nivel, con historia de dolor abdominal difuso severo de 1 año de evolución resistente a múltiples esquemas de analgésicos y emesis intermitente. Síntomas constitucionales y antecedentes de pancreatitis negativos. No hay presencia de masas o ascitis en el examen físico. El hemograma, bilirrubinas, transaminasas, fosfatasa alcalina, amilasa, lipasa y marcadores tumorales en sangre (antígeno carcinoembrionario $[\mathrm{ACE}]$ y antígeno carbohidrato $[\mathrm{Ca}]$ 19-9) fueron normales. La TAC abdominal contrastada evidenció una lesión redondeada quística septada bien delimitada en cuerpo y cola del páncreas de 54 x 40 x 60 mm (Figura 1).

Se realizó una ultrasonografía endoscópica (EUS) biliopancreática que mostró una masa sólido-quística de $60 \mathrm{x}$ $60 \mathrm{~mm}$ en la cola del páncreas, con un grosor de la pared de $4 \mathrm{~mm}$, ecogenicidad mixta, sin componente vascular ni calcificaciones; y la presencia de una lesión de aspecto polipoide en su interior de $30 \mathrm{~mm}$ (Figura 2). Se realizó una punción con aguja fina (FNA) con aguja n. $19 \mathrm{G}$ (Wilson Cook) (Figura 3) y se obtuvieron 4 cc de líquido mucoide con múltiples fragmentos blanco-amarillos, para el procesamiento de citología y bloque celular. La coloración hematoxilina-eosina mostró células epiteliales escamosas y mononucleares, tejido necrótico, hemorragia reciente y antigua con escasas células glandulares sin atipia. No había evidencia de tejido pancreático. El procesamiento del ACE no se realizó porque no había disponibilidad de dicho recurso en la institución.

Teniendo en cuenta el dolor crónico intratable, los hallazgos en la TAC, la EUS y la citología no conclusiva para malignidad, se llevó a una laparotomía para la realización de pancreatectomía distal y linfadenectomía regional. Se encontró un severo proceso inflamatorio peritumoral pancreático, por lo que fue necesaria la esplenectomía en bloque. El procedimiento no presentó complicaciones, ni requirió la trasfusión de hemoderivados. La paciente presentó una recuperación progresiva favorable, con un egreso hospitalario al décimo día. No desarrolló fistula pancreática.
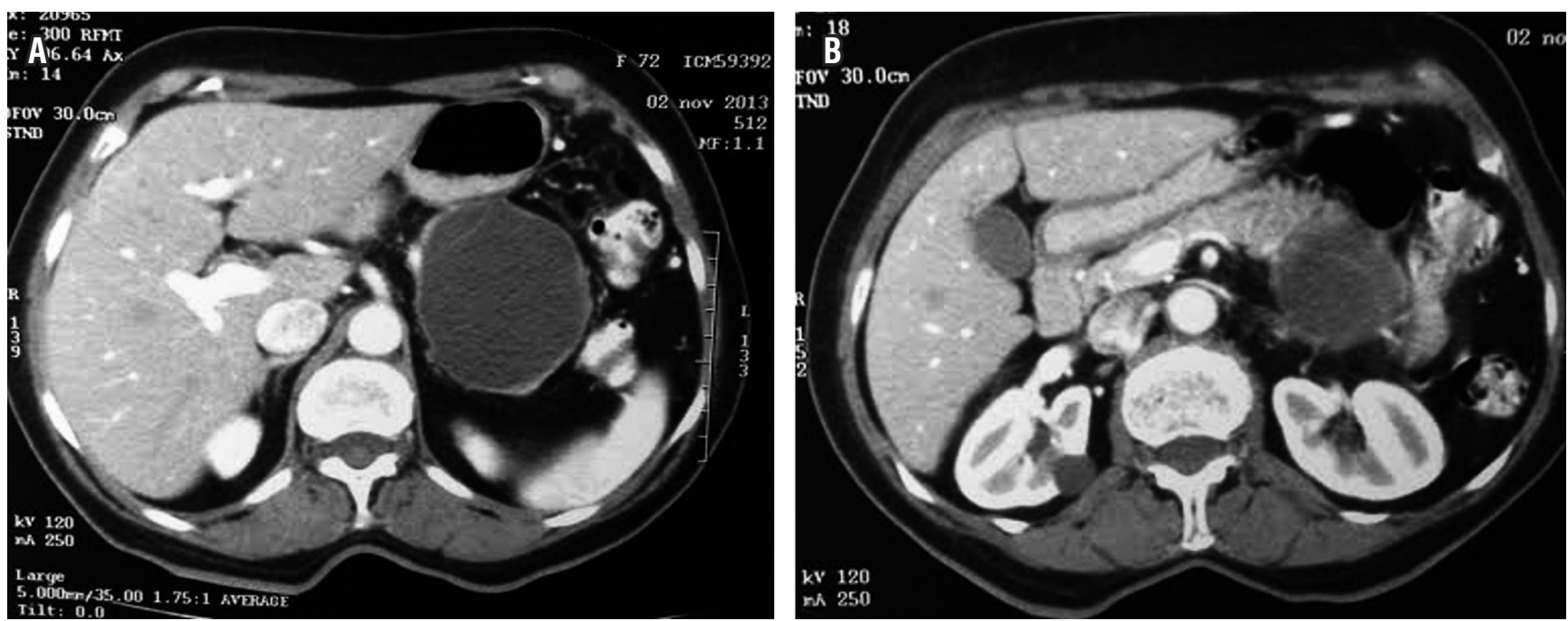

Figura 1. A. Masa quística pancreática en cuerpo y cola. B. Masa en la cola del páncreas con septos en su interior. 


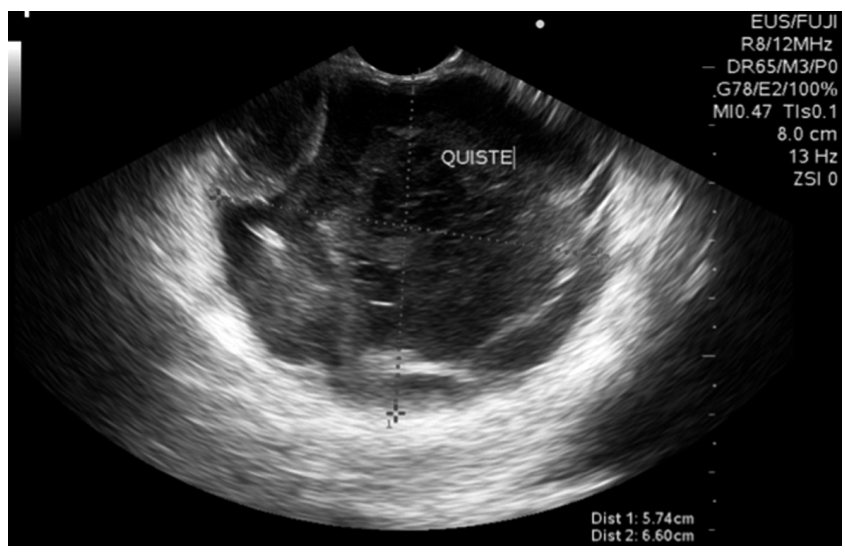

Figura 2. EUS de lesión quística en la cola del páncreas.

Se extrajo una lesión quística de 12 x 8 × $8 \mathrm{~cm}$ compuesta de un material lechoso y una lesión polipoide de $3 \mathrm{~cm}$ en su interior. Microscópicamente, la pared del quiste está revestida por células epiteliales columnares con citoplasma de aspecto mucinoso y núcleos sin atipia significativa que se rodean de un estroma de aspecto ovárico (Figura 4). Los ganglios linfáticos regionales y los bordes de resección pancreáticos no estaban comprometidos por el tumor. Los estudios de inmunohistoquímica mostraron reactividad en las células epiteliales para CA 19-9, citoqueratina 7 (Figuras 5 y 6), y reactividad en el estroma subyacente para receptores de progesterona, actina de músculo liso y desmina (Figuras 7, 8 y 9).

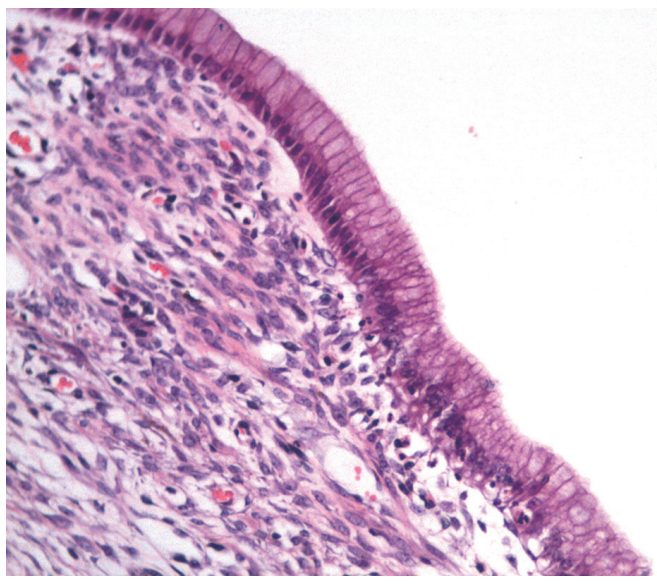

Figura 4. Microscopia (coloración de hematoxilina y eosina, 200x). Pared del quiste revestida por un epitelio columnar rodeado por un tejido estromal denso. Las células epiteliales de revestimiento presentan un citoplasma de aspecto mucinoso y núcleos sin atipia significativa. El tejido subyacente es de apariencia similar al estroma ovárico.

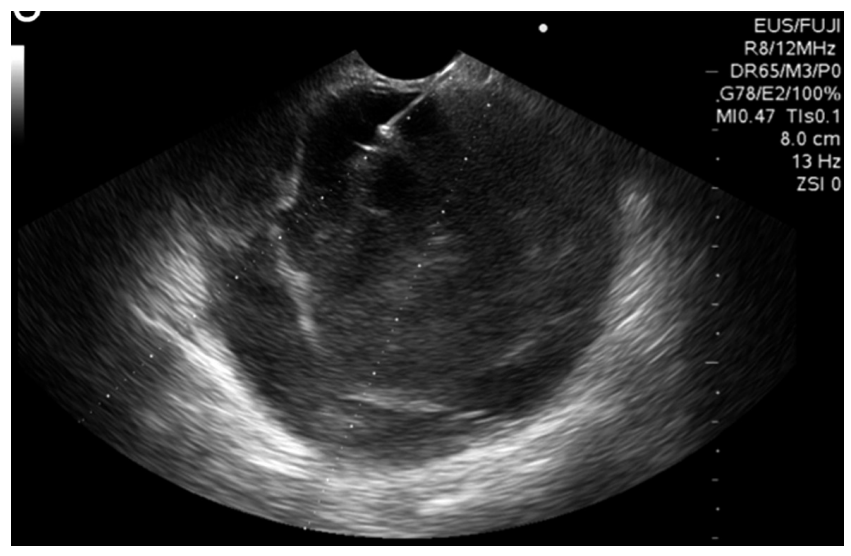

Figura 3. Obtención de líquido quístico mediante EUS-FNA.

\section{DISCUSIÓN}

Las NQM afectan de forma principal, pero no exclusiva, a mujeres en la quinta década de la vida; la relación mujer:hombre es de 20:1 (3,4); en general, son tumores únicos y se localizan en el cuerpo y la cola del páncreas, característica que les confiere una presentación clínica inespecífica, y en la mayoría de casos son asintomáticos. Pueden ser uni o multiloculares, y a menos que formen trayectos fistulosos o erosiones, normalmente no tienen comunicación con el sistema ductal pancreático. Sin embargo, un estudio multiinstitucional japonés reportó un $18 \%$ de comunicación con el conducto pancreático. Su tamaño de presen-

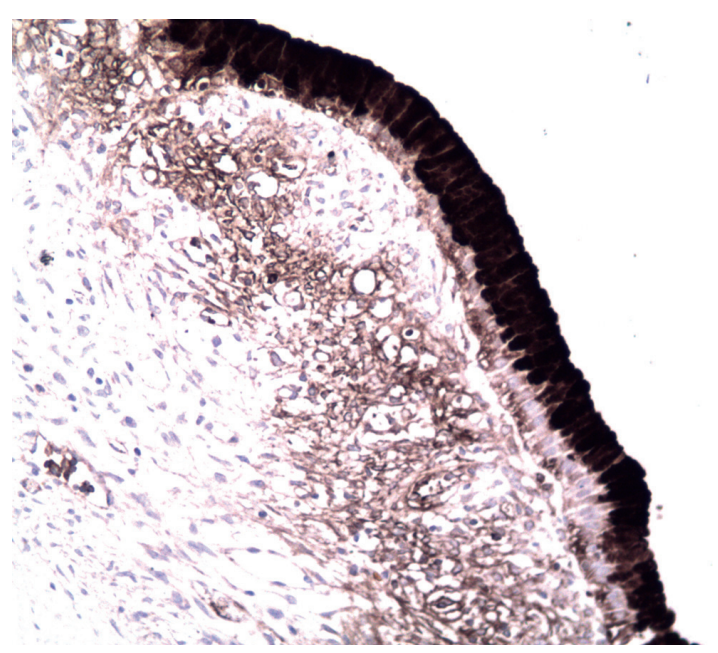

Figura 5. Microfotografía inmunohistoquímica (200x). Las células epiteliales que revisten la lesión muestran reactividad para CA 19-9. 


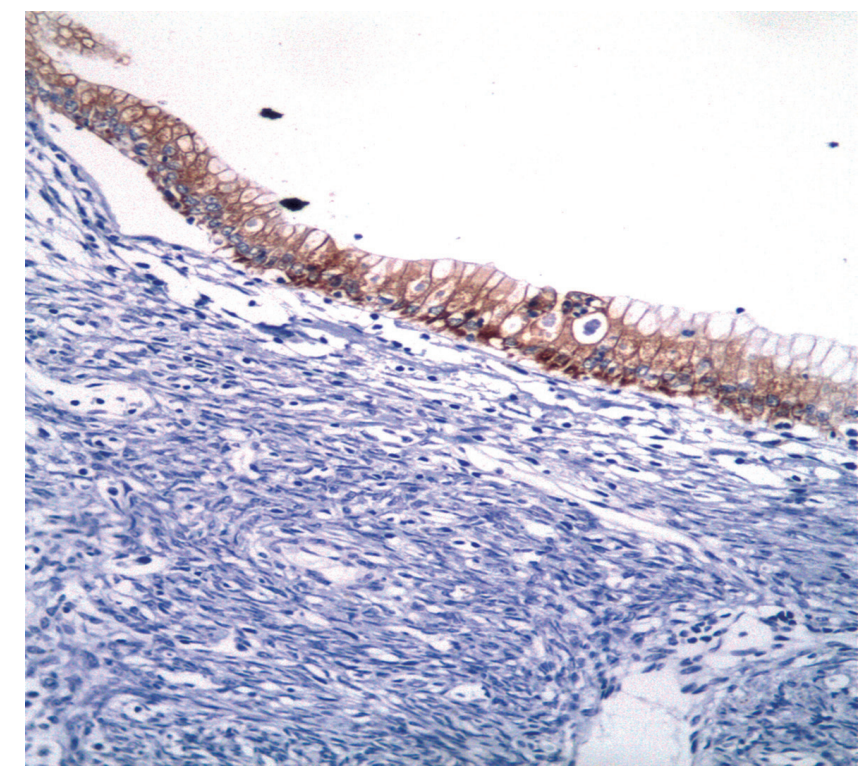

Figura 6. Microfotografía inmunohistoquímica (200x). Las células epiteliales que revisten la lesión muestran reactividad para citoqueratina 7.

tación puede ser variable, alcanzando diámetros de hasta 35 $\mathrm{cm}$. Las pruebas de laboratorio son inespecíficas.

Macroscópicamente, las NQM están rodeadas por una pared fibrosa gruesa, en ocasiones calcificada; su contenido es mucinoso o una mezcla de mucina con líquido hemorrágico o acuoso. Microscópicamente, el quiste está recubierto por células epiteliales productoras de mucina que pueden ser planas, columnares o de aspecto papilar, con diferenciación intestinal o gástrica (1). Son positivos para

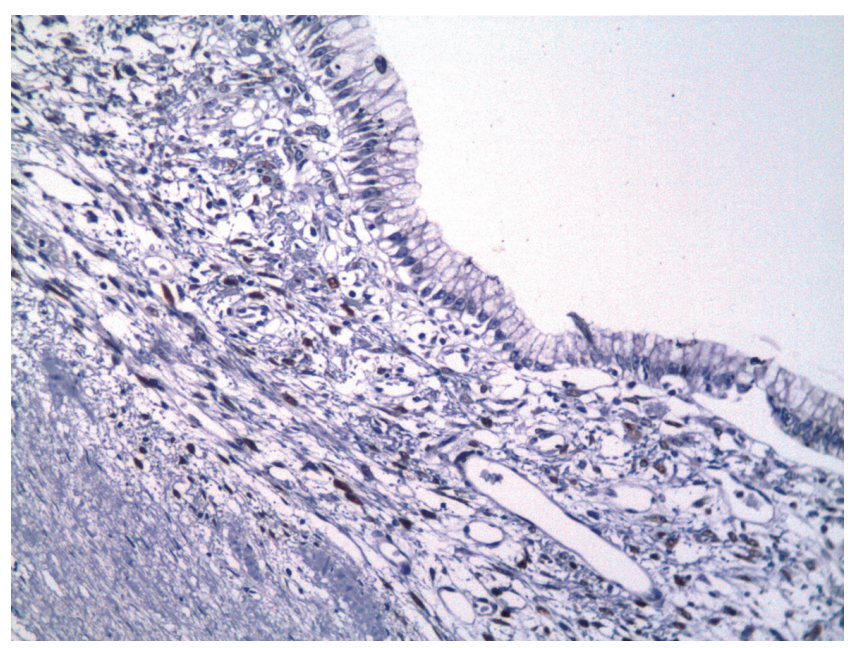

Figura 7. Microfotografía inmunohistoquímica (200x). El estroma subyacente al epitelio del revestimiento muestra reactividad para receptores de progesterona. antígeno de membrana epitelial, citoqueratinas 7, 8, 18, 19 y CA 19-9 (5). La presencia de estroma similar al ovario es característico y necesario para establecer el diagnóstico de NQM. Las células estromales pueden presentar reactividad para receptores de estrógeno y receptores de progesterona, vimentina, actina de músculo liso, desmina, calretinina, inhibina $\alpha$, melan-A, tirosina hidroxilasa, CD 99, y célula-B CLL/linfoma 2 (Bcl-2) $(1,6)$.

Hasta un tercio de los casos alberga un carcinoma invasivo. Los factores de riesgo para malignidad son lesiones grandes, asociadas con nódulos o masas, calcificaciones, pared $>2 \mathrm{~cm}$, la presencia de septos y la edad avanzada $(1,7-9)$. El cistoadenocarcinoma mucinoso es el más común; su presentación se asemeja al adenocarcinoma ductal pancreático (ADP), pero otros tipos de neoplasias pueden ocurrir, incluidos carcinomas no diferenciados con células gigantes similares a osteoclastos, carcinoma adenoescamoso, coriocarcinoma o sarcoma de alto grado. En todos los casos deben realizarse varias tomas de muestra, porque el componente invasor es pequeño y fácilmente se puede pasar por alto.

Cualquier quiste pancreático mayor de $1 \mathrm{~cm}$ debe caracterizarse por TAC de abdomen o RM abdominal con gadolinio. La apariencia tomográfíca de las NQM es variable, pueden ser similares a los pseudoquistes o los cistoadenomas serosos. Son uni o multiloculadas, y macroquísticas $(>2 \mathrm{~cm})$. El contraste realza las paredes quísticas, lo que permite identificar septos o excresencias sólidas (Figura 1 B) (1). El compromiso hepático y peritoneal y la invasión local deben descartarse (10).

La RM tiene tasas de detección de quistes más altas $(19,9 \%)$, a diferencia de la TAC $(1,2 \%-2,6 \%)$. Define con mayor precisión la relación del conducto pancreático con

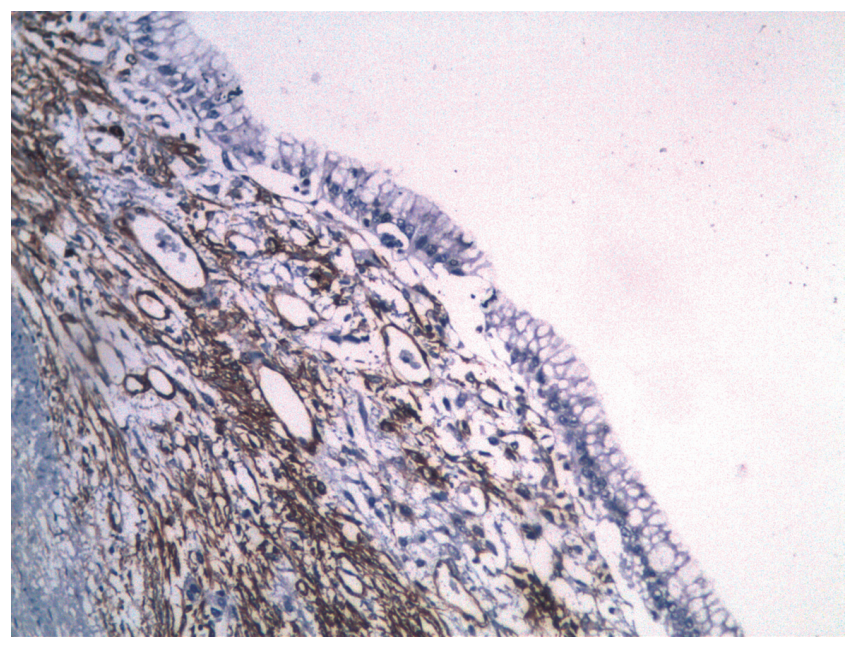

Figura 8. Microfotografía inmunohistoquímica (200x). El estroma subyacente al epitelio del revestimiento muestra reactividad para actina de músculo liso. 


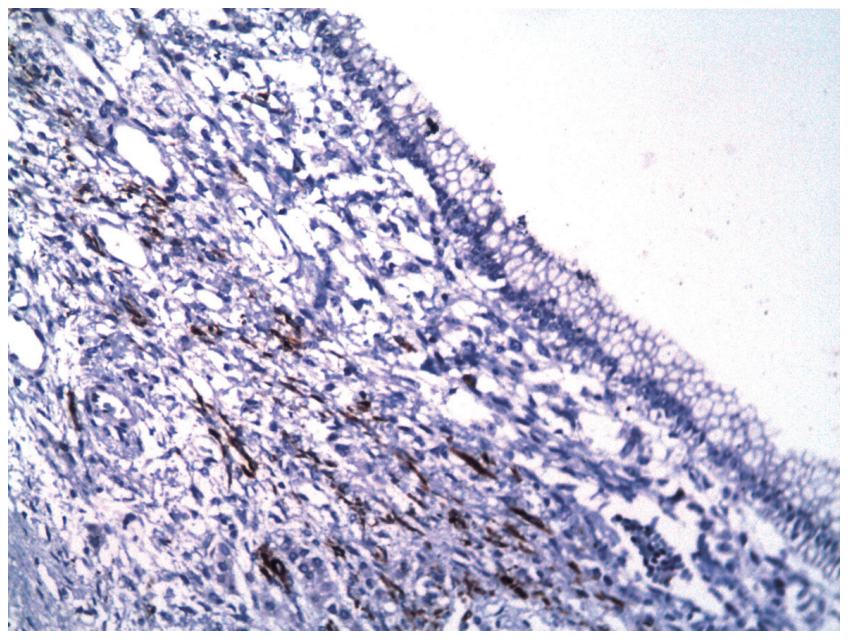

Figura 9. Microfotografía inmunohistoquímica (200x). El estroma subyacente al epitelio del revestimiento muestra reactividad para desmina.

la lesión, diferenciando los pseudoquistes o los NMPI; y caracteriza mejor el contenido del quiste en $\mathrm{T} 1$ y T2, siendo esta la principal ventaja con respecto a la TAC. Los hallazgos son similares a la TAC, pero las calcificaciones no pueden apreciarse por este método (11).

La tomografía con emisión de positrones y ${ }^{18} \mathrm{~F}$-fluorodesoxiglucosa $\left({ }^{18} \mathrm{FDG}\right)$ tienen una sensibilidad y especificidad del $94 \%$ y $97 \%$, respectivamente, en el diagnóstico de lesiones quísticas. No obstante, su papel en las NQM no ha sido bien establecido, debido a que algunos tumores no captan el ${ }^{18} \mathrm{FDG}$ (falsos negativos) (1).

La EUS es la herramienta de evaluación ideal. Tiene un bajo riesgo y permite la obtención de biopsias. Los criterios para diferenciar quistes mucinosos (septos macroquísticos o masas adyacentes) de los no mucinosos (uniloculares, panal de abejas o pared engrosada), tienen sensibilidad y especificidad bajas, $56 \%$ y $45 \%$, respectivamente; y la precisión diagnóstica es del 51\% (12). La superposición de características morfológicas entre los diferentes tipos de quistes es la razón de la baja precisión a pesar de la adecuada visualización de las estructuras (13).

La presencia de lesiones sólidas intraquísticas, irregularidad y engrosamiento de la pared o masa sólida adyacente son signos sugestivos de malignidad (2). La falta de comunicación con el conducto pancreático los diferencia de las NMPI (14).

La EUS-FNA, realizada mediante el uso de un ecoendoscopio lineal desde el duodeno o el estómago, permite obtener material citológico en tiempo real de manera segura a través del uso de agujas de 19 o 22 Gauges, dirigidas hacia la luz del quiste. Lo ideal es hacer la punción en un solo paso, aspirando el contenido hasta lograr el colapso del quiste y, de este modo, prevenir complicaciones infecciosas. Debe tomarse biopsia de los nódulos, septos, y masas adyacentes asociadas. Se debe administrar una dosis de antibiótico profiláctico y durante 3 a 5 días después de la punción para prevenir la infección (13).

El tamaño y la localización no son predictores de la aspiración exitosa del quiste. $1,5 \mathrm{~cm}$ es el tamaño requerido como mínimo para lograr tasas de éxito del 85\% (15). Esta herramienta facilita la evaluación de algunas características en el fluido del quiste, tales como la viscosidad, la citología, la química, marcadores tumorales y técnicas de análisis molecular.

La viscosidad se determina a través del signo de la cuerda y la medición de la viscosidad relativa. El primero se realiza colocando una gota del fluido obtenido entre el dedo índice y el pulgar, y se mide la longitud de estiramiento del moco antes de su disrupción. Los valores mayores de 3,5 mm se asocian con lesiones premalignas o malignas (16). El segundo se lleva a cabo mediante el uso de un viscómetro, y la utilización del agua como medida estándar (viscosidad relativa igual a 1). Los valores mayores de 1,6 $\mathrm{mm}$ permiten diferenciar lesiones quísticas mucinosas y no mucinosas con una sensibilidad del $89 \%$ y especificidad del 100\% (17).

La citología es uno de los métodos diagnósticos más precisos; sin embargo, obtener una muestra suficiente es difícil, debido al volumen limitado y baja celularidad aspirada, con una sensibilidad menor del 50\%. Los niveles de amilasa pueden elevarse en lesiones comunicadas con el sistema ductal pancreático, pero no son de utilidad en la diferenciación de quistes mucinosos y no mucinosos. Van der Waaij y colaboradores encontraron que las amilasas menores de $250 \mathrm{U} / \mathrm{L}$ son sugestivas de NQS, NQM y cistoadenocarcinomas mucinosos, con una sensibilidad de $44 \%$ y especificidad del 98\% (13).

Hammel y colaboradores describieron por primera vez la presencia de altas concentraciones de marcadores tumorales en el fluido de las NQM. Desde entonces, se han publicado múltiples estudios que evalúan el uso de los marcadores como predictores de lesiones mucinosas y malignas (ACE, CA 72-4, CA 125, CA 19-9 y CA 15-3). El ACE es el marcador más preciso en la diferenciación de lesiones quísticas mucinosas y no mucinosas. Los niveles óptimos que permiten caracterizar estas lesiones presentan gran variación, dependiendo de la serie revisada. En general, se acepta que los valores mayores o iguales a $192 \mathrm{ng} /$ $\mathrm{mL}$ tienen una precisión del $79 \%$, sensibilidad $73 \%$ y especificidad del 84\% (12). Otros marcadores tumorales se han evaluado en las lesiones quísticas, pero no han mostrado resultados satisfactorios. El análisis molecular de mutaciones KRAS, el incremento de niveles de $\mathrm{ADN}$ y la pérdida de 2 más locus en los alelos son anormalidades presentes en las neoplasias mucinosas. De todas, la mutación KRAS 
muestra la mayor especificidad (96\%), pero una sensibilidad baja (45\%). El mayor beneficio del uso de este método diagnóstico es la poca cantidad de líquido necesario para realizar el análisis: $0,2 \mathrm{~mL}$.

Los datos obtenidos producto de la punción deben analizarse con precaución, debido a que la lineabilidad, precisión, exactitud y estabilidad no se han validado. El análisis del ACE no se ha estandarizado; algunos laboratorios utilizan muestras no diluidas, mientras otros usan diluidas o mixtas. Aproximadamente, 0,5 a $1 \mathrm{~mL}$ de líquido quístico se necesita para analizar el ACE. El líquido se diluye en solución salina, se agita verticalmente para lograr homogeneidad y lo ideal es permitir que el laboratorio procese la muestra sin diluir, para obtener valores más consistentes y confiables (13). A pesar de ser la prueba de diagnóstico molecular más usada en las neoplasias quísticas pancreáticas, su interpretación es difícil sin la ayuda de información clínica adicional. Los valores dependen de las series evaluadas, así como la superposición de los resultados de ACE encontrados en los diferentes tipos de quistes (>192 ng/ $\mathrm{mL})$ en NQM y $(<5 \mathrm{ng} / \mathrm{mL})$ NQS (1). Un nivel bajo de ACE no excluye la presencia de un quiste mucinoso (18).

La tasa de complicaciones de la EUS-FNA es baja, aproximadamente $2,2 \%$; en la mayoría de casos son tempranas. La hemorragia y la infección ocurren en menos del 1\% (13).

La poca especificidad de las pruebas diagnósticas y las muestras obtenidas por biopsia, el riesgo de transformación maligna ( $6 \%$ al $27 \%)$ basado en la presencia estricta del estroma ovárico, la incapacidad de diferenciar las lesiones no invasivas de las invasivas y los buenos resultados de las resecciones quirúrgicas completas (1) permitieron que la Asociación Internacional de Pancreatología en 2010 recomendara la resección de todas las NQM. Las lesiones menores de $4 \mathrm{~cm}$ sin nódulos murales, la resección laparoscópica, resecciones preservadoras del parénquima y la pancreatectomía distal con preservación de base deben ser consideradas (18).

Entre las opciones quirúrgicas se incluyen las resecciones conservadoras de páncreas (pancreatectomía central y enucleaciones), pancreatectomías regionales (pancreatoduodenectomías y pancreatectomía distal), y pancreatectomía total. La vía de abordaje (abierta o laparoscópica) y la elección del procedimiento dependen de la localización de la lesión, las lesiones multifocales, el estado del paciente, la calidad de vida (función exocrina y endocrina), la morbimortalidad específica de cada intervención y la experiencia del cirujano.

Entre las resecciones pancreáticas segmentarias se encuentran la resección del proceso uncinado, la resección de la cabeza con preservación del duodeno, la pancreatectomía dirigida por la rama ductal y la pancreatectomía central o medial (1). Los resultados dependen de la experiencia y la curva de aprendizaje, con altas tasas de complicaciones postoperatorias, incluidas las fístulas que oscilan entre $15 \%$ y 50\% (19). Las resecciones regionales incluyen linfadenectomías, la morbilidad a los 30 días varía entre el $25 \%$ y $60 \%$, y la mortalidad entre el $2 \%$ y el $5 \%$. En el caso de las pancreatectomías distales, la tasa de fístulas oscila entre el $5 \%$ y el $20 \%(20)$.

La ablación del epitelio quístico pancreático mediante la inyección de agentes citotóxicos guiado por EUS, como el etanol (21) o paclitaxel (22), muestra resultados tempranos promisorios, aunque se requieren datos de seguimiento a largo plazo para evaluar la eficacia y determinar las indicaciones precisas para el uso de esta terapia (2).

La resección quirúrgica completa es el único tratamiento que mejora la supervivencia a largo plazo en pacientes con NQP malignas (21). La supervivencia a los 2 y 5 años de las NQM con carcinoma invasor asociado llevadas a resección quirúrgica es de $67 \%$ y $50 \%$, respectivamente (2).

La resección curativa de las NQM no invasivas no necesita ningún tipo de vigilancia postoperatoria, el seguimiento de las NQM invasivas llevadas a resección quirúrgica debe ser imagenológico (TAC/RM) cada 6 meses y no basado en la recurrencia de los síntomas (18).

\section{CONCLUSIONES}

El conocimiento de la historia natural de las NQM es incompleto y el manejo evoluciona de manera constante. La presencia de mucina es el predictor más importante de neoplasias mucinosas, seguido por los niveles de ACE $>192-200 \mathrm{ng} / \mathrm{mL}$. La evaluación de las lesiones mucinosas debe incluir características clínicas, imagenológicas, la toma de biopsias (EUS-FNA), la medición de niveles ACE y la evaluación citológica. La EUS es la herramienta de evaluación ideal y la resección quirúrgica completa es el único tratamiento que mejora la supervivencia a largo plazo.

\section{REFERENCIAS}

1. Roggin KK, Chennat J, Oto A, Noffsinger A, Briggs A, Matthews JB. Pancreatic cystic neoplasm. Curr Probl Surg. 2010;47(6):459-510. doi: 10.1067/j.cpsurg.2010.02.002.

2. Yoon WJ, Brugge WR. Pancreatic cystic neoplasms: diagnosis and management. Gastroenterol Clin North Am. 2012;41(1):103-18. doi: 10.1016/j.gtc.2011.12.016.

3. Suzuki M, Fujita N, Onodera H, Kayaba Y, Suzuki S, Kagaya $\mathrm{H}$, et al. Mucinous cystic neoplasm in a young male patient. $\mathrm{J}$ Gastroenterol. 2005;40(11):1070-4. doi: 10.1007/s00535005-1697-6.

4. Kobayashi T, Teruya M, Shimizu S, Miki K, Kobayashi K, Morita K. Mucinous cystic tumor of the pancreas in a man: a rare case. Pancreas. 2006;33(3):312-4. doi: 10.1097/01. mpa.0000229012.47291.8d. 
5. Lüttges J, Feyerabend B, Buchelt T, Pacena M, Klöppel G. The mucin profile of noninvasive and invasive mucinous cystic neoplasms of the pancreas. Am J Surg Pathol. 2002;26(4):466-71. doi: 10.1097/00000478-20020400000008.

6. Yeh MM, Tang LH, Wang S, Robert ME, Zheng W, Jain D. Inhibin expression in ovarian-type stroma in mucinous cystic neoplasms of the pancreas. Appl Immunohistochem Mol Morphol. 2004;12(2):148-52. doi: 10.1097/00129039200406000-00009.

7. Crippa S, Salvia R, Warshaw AL, Domínguez I, Bassi C, Falconi M, et al. Mucinous cystic neoplasm of the pancreas is not an aggressive entity: lessons from 163 resected patients. Ann Surg. 2008;247(4):571-9. doi: 10.1097/ SLA.0b013e31811f4449.

8. Goh BK, Tan YM, Chung YF, Chow PK, Cheow PC, Wong $\mathrm{WK}$, et al. A review of mucinous cystic neoplasms of the pancreas defined by ovarian-type stroma: clinicopathological features of 344 patients. World J Surg. 2006;30(12):223645. doi: 10.1007/s00268-006-0126-1.

9. Sarr MG, Carpenter HA, Prabhakar LP, Orchard TF, Hughes $S$, van Heerden JA, et al. Clinical and pathologic correlation of 84 mucinous cystic neoplasms of the pancreas: can one reliably differentiate benign from malignant (or premalignant) neoplasms? Ann Surg. 2000;231(2):205-12. doi: 10.1097/00000658-200002000-00009.

10. Procacci C, Carbognin G, Accordini S, Biasiutti C, Guarise A, Lombardo F, et al. CT features of malignant mucinous cystic tumors of the pancreas. Eur Radiol. 2001;11(9):162630. doi: $10.1007 / \mathrm{s} 003300100855$.

11. Minami M, Itai Y, Ohtomo K, Yoshida H, Yoshikawa K, Iio M. Cystic neoplasms of the pancreas: comparison of MR imaging with CT. Radiology. 1989;171(1):53-6. doi: 10.1148/radiology.171.1.2928546.

12. Brugge WR, Lewandrowski K, Lee-Lewandrowski E, Centeno BA, Szydlo T, Regan S, et al. Diagnosis of pancreatic cystic neoplasms: a report of the cooperative pancreatic cyst study. Gastroenterology. 2004;126(5):1330-6. doi: 10.1053/j.gastro.2004.02.013.

13. Samarasena JB, Nakai Y, Chang KJ. Endoscopic ultrasonography-guided fine-needle aspiration of pancreatic cystic lesions: a practical approach to diagnosis and management.
Gastrointest Endosc Clin N Am. 2012;22(2):169-85, vii. doi: 10.1016/j.giec.2012.04.007.

14. Hutchins GF, Draganov PV. Cystic neoplasms of the pancreas: a diagnostic challenge. World J Gastroenterol. 2009; 15(1):48-54. doi: 10.3748/wjg.15.48.

15. Walsh RM, Zuccaro G, Dumot JA, Vargo J, Biscotti CV, Hammel J, et al. Predicting success of endoscopic aspiration for suspected pancreatic cystic neoplasms. JOP. 2008;9(5):612-7.

16. Leung KK, Ross WA, Evans D, Fleming J, Lin E, Tamm EP, et al. Pancreatic cystic neoplasm: the role of cyst morphology, cyst fluid analysis, and expectant management. Ann Surg Oncol. 2009;16(10):2818-24. doi: 10.1245/s10434009-0502-9.

17. Lewandrowski KB, Southern JF, Pins MR, Compton CC, Warshaw AL. Cyst fluid analysis in the differential diagnosis of pancreatic cysts. A comparison of pseudocysts, serous cystadenomas, mucinous cystic neoplasms, and mucinous cystadenocarcinoma. Ann Surg. 1993;217(1):41-7. doi: 10.1097/00000658-199301000-00008.

18. Tanaka M, Fernández-del Castillo C, Adsay V, Chari S, Falconi M, Jang JY, et al. International consensus guidelines 2012 for the management of IPMN and MCN of the pancreas. Pancreatology. 2012;12(3):183-97. doi: 10.1016/j. pan.2012.04.004.

19. Assalia A, Gagner M. Laparoscopic pancreatic surgery for islet cell tumors of the pancreas. World J Surg. 2004;28(12):1239-47. doi: 10.1007/s00268-004-7617-8.

20. Kooby DA, Gillespie T, Bentrem D, Nakeeb A, Schmidt MC, Merchant NB, et al. Left-sided pancreatectomy: a multicenter comparison of laparoscopic and open approaches. Ann Surg. 2008;248(3):438-46. doi: 10.1097/ SLA.0b013e318185a990.

21. Oh HC, Seo DW, Lee TY, Kim JY, Lee SS, Lee SK, et al. New treatment for cystic tumors of the pancreas: EUS-guided ethanol lavage with paclitaxel injection. Gastrointest Endosc. 2008;67(4):636-42. doi: 10.1016/j.gie.2007.09.038.

22. Sharma RR, London MJ, Magenta LL, Posner MC, Roggin KK. Preemptive surgery for premalignant foregut lesions. J Gastrointest Surg. 2009;13(10):1874-87. doi: 10.1007/ s11605-009-0935-2.

23. Farrell JJ. Pancreatic cysts and guidelines. Dig Dis Sci. 2017;62(7):1827-39. doi: 10.1007/s10620-017-4571-5. 